\title{
Histopathological study of cystoscopic bladder biopsies
}

\author{
Baidya $\mathrm{R}^{1}$, Sigdel B ${ }^{1}$, Baidya $\mathrm{NL}^{2}$ \\ ${ }^{I}$ Department of Pathology, B and B Hospital, Lalitpur, Nepal. \\ ${ }^{2}$ General Surgery, B and B Hospital, Lalitpur, Nepal.
}

\section{Keywords: \\ Adenocarcinoma; Cystitis; \\ Paraganglioma; \\ Transitional cell carcinoma; \\ Tuberculosis; Urinary bladder;}

\begin{abstract}
Background: Urinary bladder diseases constitute an important source of clinical signs and symptoms which are more disabling than lethal. The prevalence of bladder tumor in developed countries is approximately six times higher compared with that in developing countries. The aim of this study was to find out various lesions in urinary bladder biopsies and its frequencies.
\end{abstract}

Materials and methods: This was a five year retrospective study from January 2008 to December 2013, carried out at B\&B Hospital, Lalitpur. All the relevant data of 324 patients who had undergone cystoscopic biopsy of urinary bladder were included in the study. All blocks were retrieved and stained with Hematoxylin and Eosin stain and examined under light microscope.

Results: The spectrum of pathological lesions included inflammations and tumors. Non-neoplastic lesions were predominant $(61.11 \%)$ followed by Transitional cell tumors accounted for 124 cases (38.27\%). Non-neoplastic lesions comprises of cystitis and tuberculosis. Papillary urothelial neoplasms was the most common tumors seen in this study with 55 low grade, and 54 high grade Papillary urothelial carcinoma according to recent WHO grading. Single cases each of adenocarcinoma and paraganglioma were diagnosed.

Conclusion: Cystoscopic biopsies help in the early diagnosis and treatment of various bladder lesions. Analysis of cystoscopic biopsies was done to ascertain the type of urinary bladder lesions in our country.

\section{INTRODUCTION}

Diseases of the urinary bladder, particularly inflammation (cystitis), constitute an important source of clinical signs

\section{Correspondence:}

Dr. Reena Baidya, $M D$

Department of Pathology,

Kantipur Dental College \& Research Center, Kathmandu, Nepal

E-mail: reenashrest@hotmail.com and symptoms. Usually, however these disorders are more disabling than lethal. Neoplasms of bladder pose biologic and clinical challenges. ${ }^{1}$ Various risk factors include cigarette smoking, industrial exposure to Acrylarume, Schistosoma Hematobium, Cyclophasphamide, artificial sweeteners and long acting useof analgesics. How these factorsinfluence to induce cancer is unclear but a number of cytogenetic and molecular alterations are heterogeneous. ${ }^{2}$

In general, the prevalence of bladder tumor in developed 
countries is approximately six times higher when compared with that in developing countries. ${ }^{3}$

This study was carried out with the aim of finding out the histopathological features of various lesions in bladder biopsies and to study them along with the frequency of different pathological lesions, particularly transitional cell carcinoma (TCC) in urinary bladder biopsies.

\section{MATERIALS AND METHODS}

This was a retrospective and prospective study done in the department of pathology at B\&B Hospital for a period of 3 years and 4 months from October 2012 to January 2015. Patients were explained and written consent was taken. Permission from the hospital institutional review committee was taken. A total of 324 cases were included in this study. Cystoscopic bladder biopsies were performed. The biopsies were preserved in $10 \%$ formalin. Gross examination was done and processed routinely. The slides were stained with Hematoxylin and Eosin stain and light microscopy technique was used for diagnosis. Data were analyzed and statistically evaluated wherever possible.

\section{RESULTS}

Out of 324 patients, $216(66.66 \%)$ were male and 108 $(33.33 \%)$ were females. The peak age of incidence was between 61-70 years (Table 1.)

The spectrum of pathological lesions included inflammations and tumors as shown in Table 2.Non-neoplastic lesions were predominant $(61.11 \%)$ followed by Transitional cell tumors accounted for 124 cases (38.27\%). Non-neoplastic lesions comprises of cystitis and tuberculosis. Papillary urothelial neoplasms was the most common tumors seen in this study with 55 low grade, and 54 high grade Papillary

Table: 1 Age and sex distribution

\begin{tabular}{cccc}
\hline Age (Years) & Male & Female & $\begin{array}{c}\text { No. of Cases } \\
\mathbf{( \% )}\end{array}$ \\
\hline $11-20$ & 4 & 3 & $7(2.16)$ \\
$21-30$ & 8 & 9 & $17(5.25)$ \\
$31-40$ & 26 & 35 & $61(18.83)$ \\
$41-50$ & 23 & 15 & $38(11.73)$ \\
$51-60$ & 36 & 19 & $55(16.98)$ \\
$61-70$ & 58 & 14 & $72(22.22)$ \\
$71-80$ & 41 & 4 & $45(13.89)$ \\
$81-100$ & 20 & 9 & $29(8.95)$ \\
Total & $\mathbf{2 1 6}$ & $\mathbf{1 0 8}$ & $\mathbf{3 2 4}(\mathbf{1 0 0})$ \\
\hline
\end{tabular}

urothelial carcinoma according to recent WHO grading (Table. 2). In this study, 1 case of adenocarcinoma and 1 case of paraganglioma were diagnosed.

\section{DISCUSSION}

In our study, maximum numbers of cases $58.95 \%$ were of chronic nonspecific cystitis. Chronic cystitis usually occurs from repeated attacks of acute cystitis. It varies greatly in severities. There may be only a mild exudate predominantly of lymphocytes and macrophages in the subepithelial tissues, or the inflammation may involve the whole thickness of the bladder wall, with an extensive exudate of lymphocytes, macrophages, and plasma cells.

In the present study, tubercular cystitis was found in $0.93 \%$ cases. Tuberculosis remains the most common cause of granulomatous inflammation of the bladder in many parts of the world. It invariably develops from the secondary foci, most often located in kidney. ${ }^{4}$ Most bladder lesions are found in the trigone, especially around the ureteral orifices. The usual tuberculous granulomata appear in the submucosa which are usually few but may become confluent, with caseous necrosis. In males, there may be secondary involvement of the prostate, whereas in the females the disease may be complicated by vasico-vaginal fistula. ${ }^{5}$

Carcinoma in situ is a neoplastic change of the urethelium considered to be a high-grade neoplasm and is an indicator of progression of urothelial neoplasm that requires specific treatment. ${ }^{6}$ In this study incidence of carcinoma in situ was $0.62 \%$.

Majority of urothelial tract tumors are epithelial. Bladder cancer is the 7th most common cancer worldwide. In general, the prevalence of bladder tumors in developed countries is approximately 6 times higher to developing countries. The most common type of bladder cancer in developed countries is urothelial carcinoma (90\%). ${ }^{3}$ In our study, TCC accounted $38.28 \%$ of all tumors while the adenocarcinoma and paraganglioma were $0.31 \%$ respectively. In Sri Lanka, TCC accounted for $93.4 \%$ of primary bladder cancer, there was a male predominance with sex ratio of $6: 1{ }^{2}$ Tumor staging and the grading are the two major factors for recurrence and progression, and for determination of treatment options for patients with bladder carcinoma. ${ }^{7}$ Histological distribution according to $\mathrm{WHO}$ grading reveals that the maximum number of cases (16.98\%) observed TCC-low grade followed by high grade TCC with $16.67 \%$. This correlates well with the study done by Matalka et al which showed $40 \%$ cases of high grade TCC and $60 \%$ of low grade TCC. ${ }^{7}$

Papillary urothelial Neoplasm of low malignant potential (PUNLMP) was seen in $4.01 \%$. jung-Weon Shiam et al showed $27.3 \%$ in his study. ${ }^{8}$ PUNLMP is a urothelial lesion that biologically carries a low risk of progression of up to 
Table 2: Histopathological diagnosis of cystoscopic bladder biopsies

\begin{tabular}{lc}
\hline \multicolumn{1}{c}{ Diagnosis } & No. of cases (\%) \\
\hline Normal Urothelium & $4(1.23)$ \\
Cystitis & $191(58.95)$ \\
Tuberculosis & $3(0.93)$ \\
Urothelial Carcinoma In-situ & $2(0.62)$ \\
Papillary Urothelial Neoplasm of Low & $13(4.01)$ \\
Malignant Potential & $54(16.67)$ \\
Papillary Urothelial Carcinoma - High Grade & $55(16.98)$ \\
Papillary Urothelial Carcinoma - Low Grade & $1(0.31)$ \\
Adenocarcinoma & $1(0.31)$ \\
Paraganglioma & $\mathbf{3 2 4 ( 1 0 0 )}$ \\
TOTAL &
\end{tabular}

$8 \%$ according to some studies.Many of these patients had tumor recurrence $(60 \%)$ and they often presented with higher grade tumor recurrence. ${ }^{9,10}$

\section{CONCLUSION}

Cystoscopic biopsies help in the early diagnosis and treatment of various bladder lesions. Analysis of cystoscopic biopsies was done to ascertain the type of urinary bladder lesions in our country.

\section{REFERENCES}

1. Vinay Kumar, Abbas AK, and Fausto N. The lower urinary tract and male genital system: Pathologic basis of disease. 7th edn. Philadelphia:Saunders; 2004.pp1026-36.

2. Hasan SM, Imtiaz F, Hasan SM. Frequency of transitional cell carcinoma in local suburban population of karachi. JLUMHS 2007;83-85.

3. Beltran AL et al. Infiltrating urothelial carcinoma. World Health Organization Classification of Tumours. Pathology \& Genetics of Tumours of the Urinary System and Male Genital Organs. IARC Press: Lyon 2004;93-109.

4. Auerbach O. The pathology of urogenital tuberculosis, Int Clin 1940;3:21-61.

5. $\mathrm{Ba}$ - Thike $\mathrm{K}$, Than - Aye, Nan - Oo. Tuberculous vesicovaginal fistula. Int J Gynaecol Obstet 1992;37:127-30.

6. Cheng L, Cheville JC, Neumann RM, et al. Natural history of urothelial dysplasia of the bladder. Am J Surg Pathol 1999;23:443-7. Crossref

7. Matalka I, Bani-Hani K, Shotar A, Bani Hani O, BaniHani I. Transitional cell carcinoma of the urinary bladder: A clinicopathological study. Singapore Med J 2008;790-4.

8. Jung-Weon Shim, Kang Su Cho, Young-Deuk Choi et al. Diagnostic algorithm for papillary urothelial tumors in the urinary bladder. Virchows Arch 2008; 452:353-362. doi: 10.1007/s00428-0080585-x

9. Samaratunga H, Makarov DV and Epstein JI. Comparison of WHO/ ISUP and WHO classification of noninvasive papillary urothelial neoplasms for risk of progression. Urology 202;60:315. http://dx.doi. org/10.1016/S0090-4295(02)01705-3

10. Fujii Y, Kawakami S, Koga F, et al. Long-term outcome of bladder papillary urothelial neoplasms of low malignant potential. BJU Int 2003;92:559-62. Crossref 